\title{
BENEFITS AND CHALLENGES OF CONTROLLING A LED AFS (ADAPTIVE FRONT-LIGHTING SYSTEM) USING FUZZY LOGIC
}

\author{
L. LUKACS ${ }^{1)^{*}}$, M. DASSANAYAKE ${ }^{1)}$, R. MAGALHAES ${ }^{2)}$, C. FONTES ${ }^{3)}$, \\ M. EMBIRUCU ${ }^{3)}$ and I. M. PEPE ${ }^{3)}$ \\ ${ }^{1)}$ Ford Motor Company, Av. do Taboao 899, Sao Bernardo do Campo, Sao Paulo 09655-900, Brazil \\ ${ }^{2}$ SENAI/CIMATEC, Av. Orlando Gomes 1845, Salvador, Bahia 41.650-010, Brazil \\ ${ }^{3}$ Universidade Federal da Bahia, Bahia 40.210-630, Brazil
}

(Received 21 December 2009; Revised 10 March 2011)

\begin{abstract}
The vehicular illumination system has undergone considerable technological advances in recent decades such as the use of a Light Emitting Diode (LED) Adaptive Front-lighting System (AFS), which represents an industry breakthrough in lighting technology and is rapidly becoming one of the most important innovative technologies around the world in the lighting community. This paper presents AFS control alternatives using fuzzy logic (types 1 and 2) to determine its operating parameters taking into consideration the road conditions in the state of São Paulo (Brazil). Fuzzy logic is a well-known extension of the conventional (Boolean) logic that enables the treatment of uncertainty present in the information through the definition of intermediary membership values between the "completely true" and the "completely false". This technique or modeling strategy is particularly important when a multi-parameter decision must be taken or the decisions are based on the human knowledge. The results show the potential of the methodology proposed and its suitability for light control providing safer nighttime driving.
\end{abstract}

KEY WORDS : Fuzzy logic, AFS, LED, Control, Lighting

\section{NOMENCLATURE}

$a_{0}, a_{1}:$ output model parameters

$\Delta \quad$ : deviation of type-1 interval fuzzy set

$\sigma \quad$ : standard deviation of type-1 Gaussian fuzzy set

$y \quad$ : current to LEDs (mA)

$V \quad:$ velocity of the vehicle velocity $(\mathrm{km} / \mathrm{h})$

$R \quad$ : radius of the curve $(\mathrm{m})$

\section{INTRODUCTION}

The car lighting system in the car market today is still based on the halogen system, see Table 1, which also foresees that halogen will predominate for the next 5 years (Stern, 2008).

Despite the appearance of steerable auxiliary headlamps in the 1940s and steerable main headlamps in the 1960s, regulations worldwide have for many years stipulated that all vehicle headlamps have to remain laterally rigidly mounted. This is no longer the case, as AFSs have changed this paradigm radically and are being developed and deployed globally at an unprecedentedly rapid pace (Neumann, 2004).

All low-beam light distributions have long been asymmetrically biased to control glare at the expense of

*Corresponding author. e-mail: 1lukacs1@ford.com seeing distance through curves and turns. The basic AFS function, called bending light, swivels the headlamp beams (or optically shifts light within the beam) in response to the driver's steering wheel movements and/or input from an onboard gyroscope. Bending light significantly increases the distance reach of the low beam in curves, without increased glare to oncoming traffic (Ishiguro and Yamada, 2004).

The AFS approach which has given rise to most commercial interest is curve lighting. It involves in some embodiments only horizontal or vertical displacements of a given base beam pattern. Specifically, in these embodiments, curve lighting (also called bending or swiveling light) involves controlling the horizontal aim of the beam pattern (or a part of it) depending on variables such as the radius of the curve and the speed of the vehicle. There are several possible strategies for implementing curved lighting, including moving both lamps in tandem, moving both lamps but each to a different extent, moving only one of the lamps, moving lamps differentially on left and right curves,

Table 1. Global market prevalence of halogen headlamps, 2007-2013 (fleet percentage).

\begin{tabular}{cccccccc}
\hline & 2007 & 2008 & 2009 & 2010 & 2011 & 2012 & 2013 \\
\hline Prevalence & 84 & 84 & 83 & 83 & 80 & 79 & 77 \\
\hline
\end{tabular}

Source: Just-Auto, industry estimates (Just Auto, 2007) 
or energizing additional lamps (Hamm, 2002; Hogrefe, 2000; Löwenau et al., 2001).

Globally there has been a huge increase in the use of LEDs due to their low current consumption and long mean time between failures (high MTBF). HID (high-intensity discharge) headlamp burners produce between 2,800 and 3,500 lumens from between 35 and 38 watts of electrical power, while halogen filament headlamp bulbs produce between 700 and 2,100 lumens from between 40 and 72 watts at $12.8 \mathrm{~V}$ (UNECE, 2008). The first seriesproduction LED headlamps were factory-installed on the Lexus LS 600h / LS 600h L starting with the 2008 models. Low beam, front position light and sidemarkers are performed by LEDs; high beam and turn signal functions use filament bulbs. The headlamp is supplied by Koito. Full-LED headlamps supplied by AL-Automotive Lighting were fitted on the 2008 V10 Audi R8 sports car except in North America. The Hella headlamps on the 2009 Cadillac Escalade Platinum became the first U.S. market all-LED headlamps. Present designs perform between halogen and HID headlamps (Hella, 2009), with system power consumption slightly lower than other headlamps, longer life spans and more flexible design possibilities (Hella, 2009; AL, 2009). As LED technology continues to evolve, the performance of LED headlamps is predicted to improve to approach, meet, and perhaps one day surpass that of HID headlamps. Furthermore, the application of LED technology can significantly contribute to reducing $\mathrm{CO} 2$ emissions and fuel consumption.

This aspect gains particular importance with the implementation of daytime running light (DRL). DRL with LED will require $14 \mathrm{~W}$ of energy, whereas the use of conventional vehicle lighting during the day (low beam, rear lights, position lights) consumes $150 \mathrm{~W}$ (AL, 2009). Table 2 presents the trend for LED implementation in some regions of the world and it is important to mention that the emerging markets will grow further in the next 5 years (Zukauskas et al., 2002; Stern, 2008).

The main purpose of this paper is to describe the development and application of a fuzzy algorithm system based upon the specialist expertise to control an array of LEDs. Simulation tests using real data of speed and curvature radius (geometric features of the track) show that the model provides an increase in light on the curves

Table 2. Global market value by region for automotive LEDs, 2007-2013 (US\$m).

\begin{tabular}{lccccccc}
\hline Region & 2007 & 2008 & 2009 & 2010 & 2011 & 2012 & 2013 \\
\hline North America & 123.7 & 126.6 & 128.7 & 130.1 & 134.3 & 136.6 & 137.5 \\
Europe & 116.0 & 119.7 & 122.6 & 126.3 & 128.2 & 134.7 & 143.1 \\
Japan & 75.6 & 76.9 & 78.3 & 79.2 & 81.0 & 81.2 & 83.2 \\
Rest of world & 7.7 & 7.7 & 7.9 & 8.0 & 8.2 & 8.3 & 8.4 \\
\hline
\end{tabular}

Source: Just-Auto, industry estimates (Just Auto, 2007) according to the road and driver's needs making nighttime driving safer (Gotoh and Aoki, 1996). This system adds light to the beam pattern which is different from the bending light approach which does not add light to the night drive, but swivels it. The aspects associated to the fuzzy modeling are presented (section 2) as well as the device decision and controlling template (section 3 ). Some models based on fuzzy logic (types 1 and 2) were developed and applied to the LED control. The rules were defined according to specialist knowledge (section 4). The specialist, who is an expert in the automotive lighting field with more than 15 years of experience developing lighting systems in the automobile industry, understands the environment (city, motorway and rural roads) and road conditions such as pedestrians, fog and rain, for example, in the region where the model is used. As a reference, Schmocker et al. (2008) carried out a study regarding the fuzzy impact of traffic control on an urban area. He demonstrated that expert knowledge can have a huge impact on the process of modeling the rules in order to optimize the traffic around the city. The main function will be determining the initial model. Here, the specialist will have an impact on determining the initial model. Further in section 4 , the model parameters (i.e. membership function parameters associated to the consequents of each rule) were estimated through the comparison between the specialist responses (desired responses) and the model results, using data related to driver and road conditions of the South American target customer (Wördenweber et al., 2007). This procedure has enabled the selection of parameter values that can provide optimized solutions, considering that the expert's knowledge may not always involve practical rule-of-thumb guidelines concerning the appropriate estimation of these parameters. In sections 5 and 6 results and concluding remarks are presented and discussed, respectively.

\section{FUZZY KNOWLEDGE BASE AND MEMBERSHIP FUNCTIONS}

Fuzzy logic is a superset of conventional (Boolean) logic that has been extended to handle the concept of partial truth - truth values between "completely true" and "completely false", particularly important when a multi-parameter decision must be taken (Bellman and Zadeh, 1970). The most important feature of fuzzy control is that its internal mathematical model is built-up directly through expertise from human reasoning which can be expressed by a set of heuristic rules which are quantified according to fuzzy set theory. In this sense, fuzzy control algorithms and fuzzy inference systems present many advantages (Altinten et al., 2003) such as simplicity, robustness, no need to find transfer functions, nonlinear behavior and adaptability.

A type-1 fuzzy set is a generalization of a crisp set or zero-one membership function (Mendel, 2001). A crisp set $A$, denoted by $\mu_{A}(x)$, is such that: 
$A=\mu_{A}(x)=\left\{\begin{array}{lll}1 & \text { if } & x \in A \\ 1 & \text { if } & x \notin A\end{array}\right.$

A type-1 fuzzy set $F$ is defined in a universe of discourse $X$ (the range of possible values for the variable $\mathrm{x}$ ) and its membership function, denoted by $\mu_{F}(x)$, takes on values in the interval $[0,1]$. A membership function provides a measure of degree of similarity of an element in $X$ to the fuzzy set $F$. The rules of a linguistic fuzzy model have the general form:

If $x$ is $A$ then $y$ is $B$

The proposition " $x$ is $A$ " is the antecedent of the rule, and the proposition " $y$ is B" is the consequent. Variables $x$ and $y$ are linguistic ones defined as fuzzy sets in domains (universe of discourses) $\mathrm{X}$ and $\mathrm{Y}$, respectively. Constants $\mathrm{A}$ and $B$ are linguistic terms usually associated with meanings for the linguistic variables, such as "low temperature", "high velocity", etc. The rule base together with the database forms the knowledge base of the fuzzy system (Babuška and Verbruggen, 1996; Chen and Liu, 2004). The input-output mapping is accomplished by the fuzzy inference mechanism. The main approaches in this case are the Mamdani and Takagi-Sugeno-Kang (TSK) models (Liang and Mendel, 2000). The Mamdani model has fuzzy sets in the consequents, while in the TSK model the consequents are parametric models of the inputs (Mendel, 2001).

Type-2 fuzzy logic was introduced by Zadeh (1975) as an extension of traditional fuzzy logic. Subsequently, several researchers have presented and developed important contributions such as the works of Mizumoto and Tanaka (1976, 1981), Dubois and Prade (1978, 1979), Hisdal (1981) and Karnik and Mendel (1998, 2000). The origin of type-2 fuzzy logic is related to the inability of traditional fuzzy logic to deal with the uncertainty associated to the information completely (Mendel, 2003). In a type- 1 fuzzy set, the membership grade of a variable $x$ ( $x \in X$, where $X$ is the universe of discourse), is represented by $\mu_{A}(x)$, which is only a crisp number in $[0,1]$. A type- 2 fuzzy set $\tilde{A}$ has a membership grade of $x(x \in X)$ in $\tilde{A}$ represented as $\mu_{\tilde{A}}(x)$ which is a type- 1 fuzzy set in $[0,1]$. Therefore, in a type- 2 fuzzy set, an element of its domain does not have a single value as membership degree. The element is associated with another membership function (Karnik and Mendel, 1998) that represents the uncertainty related to the definition of the membership degree associated to a specific value of the linguistic variable $\mathrm{x}$.

A generic rule in a type- 2 fuzzy model has the same form presented in (2). The differences between these techniques are centered in the fuzzy sets definition. For the specific case of TSK model, the general structure for a type- 2 fuzzy model comprises the use of type- 2 fuzzy sets in the antecedents together with type-1 fuzzy sets to represent the consequent parameter. Therefore, these rules simultaneously account for the uncertainty about antecedent membership functions and consequent parameter values (Mendel, 2001). Specifically, the first is usually associated with a situation in which more than one specialist suggests different membership functions for each of the inputs (Hagras, 2004; Tahayori et al., 2006). Moreover, special cases occur when the antecedents are of type- 2 fuzzy sets but the consequents are crisp numbers and when both antecedents and consequents are type-1 fuzzy sets (Mendel, 2001).

Despite the fact that type-1 fuzzy logic has been used in several production processes since the 1970s (Østergaard, 1977; King and Mamdani, 1977) and that fuzzy systems are universal function approximations (Sala et al., 2005), many areas of human knowledge have also used type-2 fuzzy inference systems (Li et al., 2006; Pareek and Kar, 2006; Ren et al., 2007; Mendoza et al., 2007; Thovutikul et al., 2007; Du and Zhu, 2006). There are some applications of fuzzy logic in traffic control in the cities (Anderson et al., 1998; Niittymäki and Turunen, 2003; Kosonen, 2003). Anderson et al. (1998) investigated the implementation of a traffic signal controller based on a fuzzy inference system that uses travel times and emissions as inputs. A meaningful controller's sensitivity to changes in the membership function parameters was verified and a multi-objective optimization technique, based on the genetic algorithm, was investigated to minimize simultaneously all the performance measurements established. Niittymäki and Turunen (2003) studied the relation between traditional fuzzy inference system and the many-valued logic framework which are similar to classical logic because they accept the principle of truth-functionality, namely, that the truth of a compound sentence is determined by the truth values of its component sentences, in real systems of traffic signal control. The results indicated that there is a strong relationship between these two types of approach. Kosonen (2003) presents a traffic signal control system based on real-time simulation, multi-agent control scheme, and fuzzy inference. This system is closely related to the microscopic traffic simulator HUTSIM (Helsinki University of Technology Simulator) both developed by the Helsinki University of Technology. The HUTSIM simulation model is used both for off-line evaluation of the signal control scheme and for on-line simulation of specific traffic conditions under control. In the control strategy presented by Kosonen (2003) each traffic signal operates individually as an agent and the decision making of the agents is based on a fuzzy inference that considers the combination of various aspects such as fluency, economy, environment and safety. In the automotive industry, there are some other examples of fuzzy application (Hwang and Song, 2005; Feng et al., 2003; Yu et al., 2002). Hwang and Song (2005) developed a fuzzy model to estimate the vehicle's speed using the acceleration and the split ratios of the wheel as inputs. In another automotive application Yu et al. (2002) propose a new control strategy for active vehicle suspension systems by using a combined control scheme, i.e. using a genetic 
algorithm (GA) based on self-tuning PID (proportionalintegral-derivative) controller and a fuzzy controller. In this control scheme, the PID controller is used to minimize vehicle body vertical acceleration and a fuzzy controller is used to minimize the pitch acceleration. Feng et al. (2003) adopted a fuzzy approach to maintain the optimal target slip ratio in order to achieve the best compromise between braking deceleration, stopping distance and direction stability performances for the vehicle. Nevertheless, it is important to highlight that so far there is no literature available regarding the application of fuzzy logic in the automotive lighting field, more specifically regarding the control of an AFS LED system.

\section{LIGHTING DEVICE AND HARDWARE PROPOSE}

Throughout the entire automotive industry the lighting system has always played a very important role. In the past 50 years, vehicular lighting has further gained importance due to its close relationship with enhancing passenger and vehicle security. Naturally, this interwoven scenario affects both the design and how the best technical solution can be aptly translated into the vehicle's "eyes" while maintaining the brand identity, a key aspect of every vehicle.

Several technological advances have been introduced over the years, such as double beam systems (one for low and one for high beam displacements in the 1950s) and the introduction of xenon headlights in the 1970s. Although xenon technology has been available for more than 20 years, it represents no more than $20 \%$ of the entire worldwide market because of its high cost and regulatory implications on Economic Commission for Europe (ECE) markets (auto leveling and washer system). In the late 1990s the use of infrared technology along with state-of-the-art reflectors have helped drivers to experience a better night drive than before. Recently there has been much change inside the lighting community due to the appearance of relatively cheaper technology, specific customer requests and higher market expectations. The use of LEDs has led this "trend" around the world. However, due to cost and heat management issues its overall performance has not yet reached the xenon headlights level (Neumann, 2006).

When examining the actual trend from the technology and design perspective, it is very important to highlight the next generation of adaptive front lighting system (AFS) which features sequential LED lighting. The way in which the optics in this system have been designed to function together has not yet been seen or appreciated in the exterior lighting world. In addition to being a beautifully designed optical arrangement, the system also helps drivers take corners and curves more safely and consumes less energy while doing so due to the LED usage (Sivak et al., 2005).

The system senses when the vehicle is approaching a curve and directs the row of LEDs to switch on sequentially. As the vehicle turns, the LEDs illuminate at a

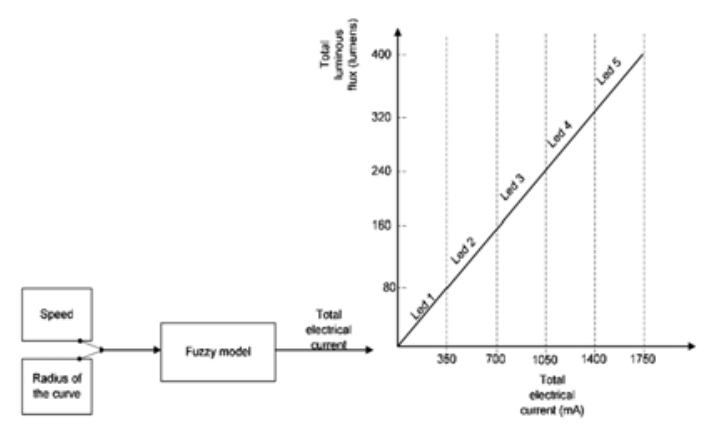

Figure 1. System configuration.

rate and intensity determined by the degree and speed of the turn. Electronic sensors analyze inputs from the steering wheel and the vehicle speed to determine how and when to illuminate the LEDs. The LEDs automatically switch off when the road straightens out, but the main beam continues to illuminate the overall road. The way the system works is shown in Figure 1. The total amount of luminous flux is linearly correlated with the total current established by the fuzzy model. Each increment of $350 \mathrm{~mA}$ in the light current totals one LED and the LEDs are activated in a sequential order. As regards the algorithm, the TSK model was adopted and two variables are considered in the antecedents: the curve radius and the vehicle speed. The output of the fuzzy inference model establishes the light intensity (total electrical current) to be added to the array of LEDs. Assuming that the light intensity is distributed progressively through the LEDs, the definition of which LED will be lit is directly related to the total current value calculated by the model.

The Birdseye view is a great tool to highlight the improvement that can be obtained with AFS. It shows the light distribution (width, length and intensity) on the road from the top view. In Figure 2 the light distribution is shown with its aperture and range. This is an existing headlamp low beam distribution with the increase in light shown in the yellow stripes added to the distribution. The range is almost 150 meters and the aperture is 40 meters. Furthermore, the light intensity varies from red (more intense) to blue (less intense) and the homogeneity of this light distribution can be perceived through the color shift from red to blue. The result with AFS is lighter, more

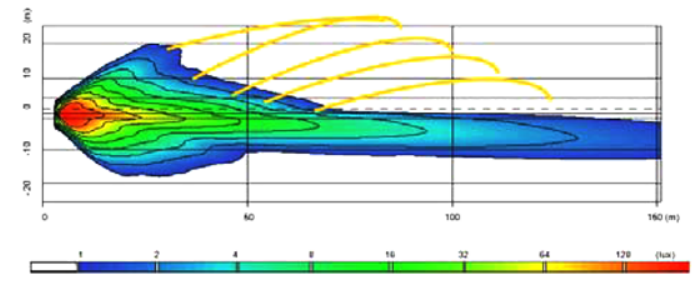

Figure 2. Birdseye view and the improvement obtained with AFS: the light (yellow) is added to the beam pattern. 
Table 3. Improved illumination by AFS.

\begin{tabular}{cccc}
\hline \multicolumn{2}{c}{$\begin{array}{c}\text { Road scenario and speed } \\
\text { conditions }\end{array}$} & \multicolumn{2}{c}{ Luminous flux ratio } \\
\hline Speed $(\mathrm{km} / \mathrm{h})$ & $\begin{array}{c}\text { Road curvature } \\
\text { radius (m) }\end{array}$ & $\begin{array}{c}\text { Without } \\
\text { AFS }\end{array}$ & With AFS \\
\hline 40 & 50 & 1 & 3.9 \\
70 & 150 & 1 & 2.8 \\
90 & 280 & 1 & 1.6 \\
\hline
\end{tabular}

accurately placed, and more importantly, light is being added (Figure 2) for more visibility during night time driving, not taken from one spot on the road and having it moved to another, as today's cornering systems do (Hara et al., 2001).

The concept that will be investigated in this study is the use of the fuzzy model in a device to control five LEDs to improve visibility for night-time driving.

\section{METHODOLOGY}

As mentioned in the previous section, the strategy adopted was to measure the road curvature and vehicle speed directly from the vehicle to determine the lighting level of each LED. For a vehicle with AFS, there is a strong relationship between these inputs and the luminous flux (Hogrefe, 2000; Ishiguro and Yamada, 2004). Table 3 shows the measured data where there is a relationship between these input variables with the luminous flux. It shows that there is significant interaction between low speed and wide curvatures. This data was acquired from a series of vehicle level evaluations at the Ford test track in Dearborn, Michigan. In these conditions in particular there is an improvement in the luminous flux obtained between vehicles with the AFS system and without AFS.

This study will analyze the impact of the implementation of fuzzy type 1 or 2 to control the AFS and compare with the specialist criteria. There are several methods to investigate the luminance and measurements on the road. For example, the quantity and the quality of road lighting can be analyzed and controlled with road surface luminance measurements. As a reference, the European standard for road lighting calculations EN 13201-3 (EN, 2003) describes methods for luminance calculations and measurements (Ekriasa et al., 2008).

The evaluation of the fuzzy inference system was carried out through a comparison between its prediction and the specialist response, using the measurements collected from the test track presented in Figure 3 (São Paulo, Brazil). This track reproduces several different driving conditions, varying speed and curves simulating the highway and city driving conditions. This test track records the road condition and driver's behavior. The data acquisition was done by a VBOX III device installed inside the vehicle. It

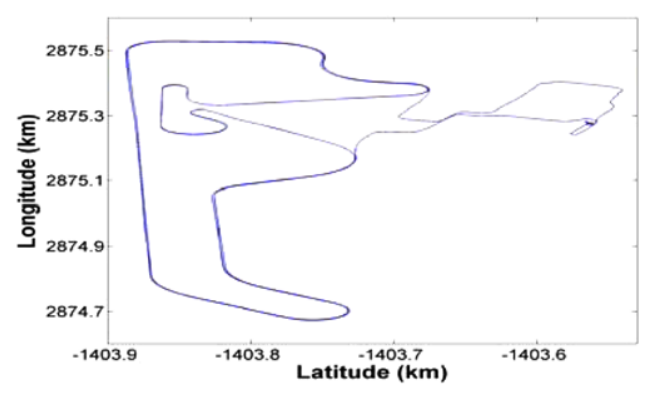

Figure 3. Test track.

features a powerful engine capable of providing $100 \mathrm{~Hz}$ update rate of all positioning parameters including velocity, heading and position (latitude and longitude). Velocity and heading data are calculated from Doppler shift in the positioning carrier signal to provide high accuracy. It allows the acquisition of fifty measurements per second, with each measurement comprising both speed and position (longitude and latitude). The longitude and latitude are used directly to calculate the radius. This approach enables useful information before, during and after the curve entrance. The data was acquired from the test track during a 4 period lap, which represents approximately 1,000 seconds.

The fuzzy inference system comprises the vehicle's speed $(\mathrm{km} / \mathrm{h})$ and curvature radius $(\mathrm{m})$ as inputs and the output is the electric current applied to drive the array of LEDs. The universes of discourse adopted for the speed and the curvature radius were $[0 ; 1000]$ and $[0 ; 140]$, respectively, which incorporates all possible values of speeds and all curve shapes. In the simulation, a comprehensible representation of the system structure is

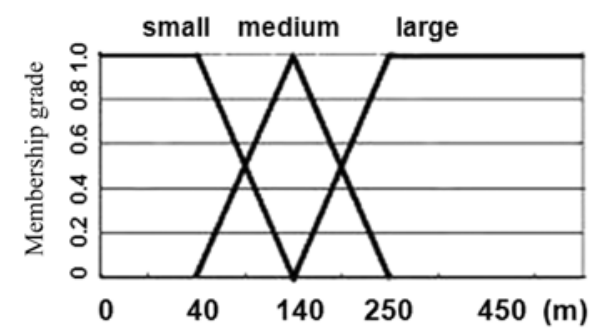

(a)

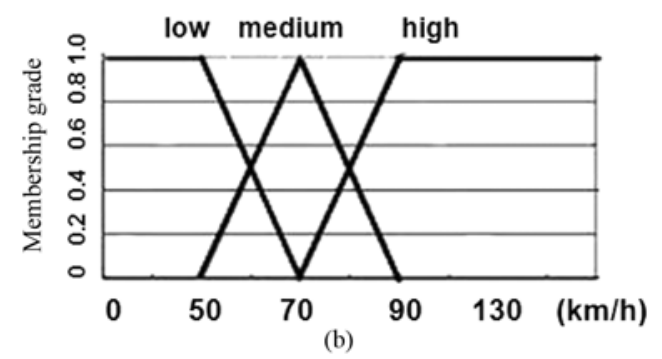

Figure 4. Membership functions related to the radius (a) and to the speed (b). 


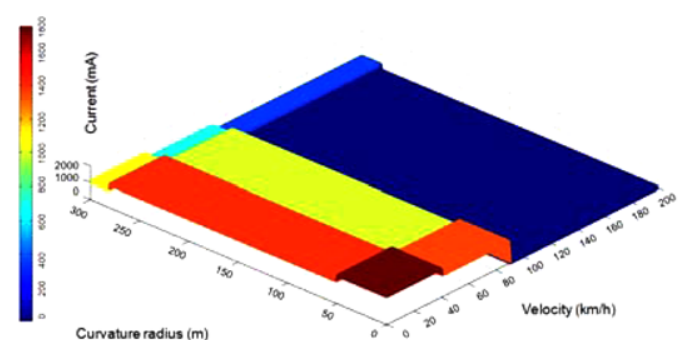

Figure 5. Surface generated from the specialist (Table 4).

adopted to investigate the model performance. Each input variable (linguistic variable) features three fuzzy sets that represent three meanings or linguistic terms: small, medium and high. All the membership functions considered to represent these fuzzy sets are shown in Figure 4 and were proposed by the specialist and these do not change during the model adjustment procedure. Although this model was developed with data from a test track, it is suitable for all road and environment conditions, such as freeways, highways and city traffic.

This work comprises the development and testing of some models, structured according to type- 1 and type- 2 fuzzy logic, based on human specialist knowledge. This knowledge base is presented in Table 4 which shows a value for the electrical current to the LEDs, according to the specialist, for each possible combination between the input ranges also defined by the specialist. The combination between high speed (speed $>90 \mathrm{~km} / \mathrm{h}$ ) and small radius (radius $<50 \mathrm{~m}$ ) was not considered due to the fact that this would lead to the vehicle crashing. In addition, Figure 5 presents the output (current to LED), according to Table 4, for each pair of inputs (speed and radius) collected on the test track shown in Figure 4. According to Figure 5, the rules of Table 4 comprise a model that presents discontinuity in the output (current value) and does not have parallel processing (only one rule will be active and will be considered for each set of values of speed and radius). The rules and values suggested by the specialist are an attempt to identify or quantify the ideal visual comfort for each situation. According to the specialist, when the vehicle goes above $90 \mathrm{~km} / \mathrm{h}$ with curvatures below $280 \mathrm{~m}$, there is no additional light to the light distribution and this might lead to a discomfort issue during night-time driving. Instead of only using the rules presented in Table 4, the fuzzy model approach can provide ways to improve the light control performance using as knowledge base the rules and membership functions presented in Table 4 and Figure 4, respectively.

The modeling of this problem using a fuzzy structure enables the construction of a model that provides continuous values for the electrical current and is also capable of considering the uncertainty associated to the definition of the membership grade of radius and speed values with relation to each range (or set) defined by the
Table 4. Antecedent combination and desired output (set by human specialist) - based on the needs of the driver.

\begin{tabular}{|c|c|c|}
\hline \# & $\begin{array}{l}\text { Rules for the antecedents } \\
\text { (specialist) }\end{array}$ & $\begin{array}{l}\text { Luminous } \\
\text { flux } \\
\text { (lumens) } \\
\text { (specialist) }\end{array}$ \\
\hline 1 & If speed $<40 \mathrm{~km} / \mathrm{h}$ and radius $<50 \mathrm{~m}$ & 400 \\
\hline 2 & $\begin{array}{l}\text { If speed }<40 \mathrm{~km} / \mathrm{h} \text { and } 50 \mathrm{~m}<\text { radius }< \\
280 \mathrm{~m}\end{array}$ & 340 \\
\hline 3 & If speed $<40 \mathrm{~km} / \mathrm{h}$ and radius $>280 \mathrm{~m}$ & 250 \\
\hline 4 & $\begin{array}{l}\text { If } 40 \mathrm{~km} / \mathrm{h}<\text { speed }<90 \mathrm{~km} / \mathrm{h} \text { and radius } \\
<50 \mathrm{~m}\end{array}$ & 320 \\
\hline 5 & $\begin{array}{l}\text { If } 40 \mathrm{~km} / \mathrm{h}<\text { speed }<90 \mathrm{~km} / \mathrm{h} \text { and } 50 \mathrm{~m} \\
<\text { radius }<280 \mathrm{~m}\end{array}$ & 240 \\
\hline 6 & $\begin{array}{l}\text { If } 40 \mathrm{~km} / \mathrm{h}<\text { speed }<90 \mathrm{~km} / \mathrm{h} \text { and radius } \\
>280 \mathrm{~m}\end{array}$ & 160 \\
\hline 7 & If speed $>90 \mathrm{~km} / \mathrm{h}$ and radius $>280 \mathrm{~m}$ & 80 \\
\hline 8 & $\begin{array}{l}\text { If speed }>90 \mathrm{~km} / \mathrm{h} \text { and } 50 \mathrm{~m}<\text { radius }< \\
280 \mathrm{~m}\end{array}$ & 0 \\
\hline
\end{tabular}

Table 5. Fuzzy model structure.

\begin{tabular}{l}
\hline$\# \quad$ Antecedents of Fuzzy Rules \\
\hline 1 If speed is low and Radius is large then $y=f_{1}(V, R)$ \\
2 If speed is low and Radius is medium then $y=f_{2}(V, R)$ \\
3 If speed is low and Radius is small then $y=f_{3}(V, R)$ \\
4 If speed is medium and Radius is large then $y=f_{4}(V, R)$ \\
5 If speed is medium and Radius is medium then $y=f_{5}(V, R)$ \\
6 If speed is medium and Radius is small then $y=f_{6}(V, R)$ \\
7 If speed is high and Radius is large then $y=f_{7}(V, R)$ \\
8 If speed is high and Radius is medium then $y=f_{8}(V, R)$ \\
\hline
\end{tabular}

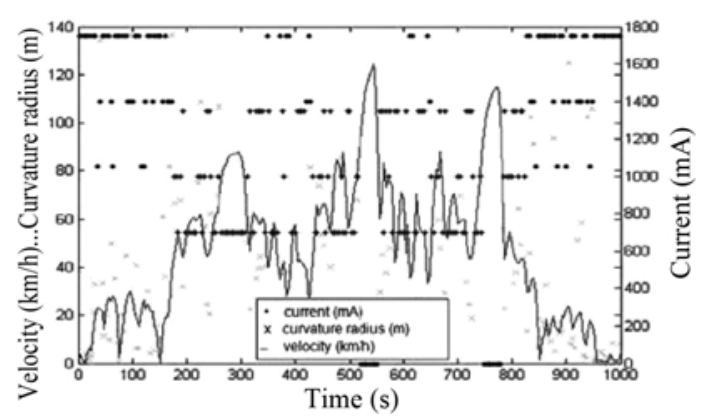

Figure 6. Current values according to the specialist.

specialist. Based on Table 4, Table 5 presents the general structure adopted in this work for the TSK fuzzy model. The antecedent of each rule was proposed directly from the knowledge in Table 4 together with the membership functions presented in Figure 4, also defined by the 
specialist. The general output model of each rule is also shown in Table 5. However, specific output models were tested and analyzed and the results are presented and discussed in section 5 .

All the model structures considered in this work were based on the TSK inference model in such a way that the electric current value in each rule is related directly with the input values through a linear parametric model. In order to adjust the fuzzy model performance, some parameters presented in the rule consequents were estimated through the solution of an optimization problem that minimizes the deviations between the model predictions and the desired current value defined by the specialist. This parameter estimation procedure keeps the intrinsic features of the fuzzy models tested and enables these models to adhere to or approximate the specialist knowledge. The type-2 TSK model proposed in this work comprises type-1 fuzzy sets in the antecedents according to the membership functions presented in the Figure 6 and type-1 fuzzy sets to represent some consequent parameters. Two aspects supported the choice of this approach in type-2 fuzzy modeling. First, the membership functions of radius and speed were defined by only one specialist according to Figure 4 and uncertainties about these sets are not meaningful to justify the adoption of type-2 fuzzy sets in the antecedents (inputs). Second, possible uncertainties in the model definition tend to predominate in the output of each rule and type-1 fuzzy sets were adopted in the consequent parameters in order to represent this uncertainty inherent to the definition of the light intensity (total electrical current) in each rule.

\section{RESULTS AND DISCUSSION}

The simulation tests comprised the performance analysis of five fuzzy model structures that differ only in regard to the consequent model considered. Table 6 presents the output models tested, the sum of squared errors (SSE) between the model results and the specialist responses in all data set and the standard deviation. Percentages of average standard deviation error and of the average quadratic error for each output model are also presented. Model 1 is a zero order TSK model of type-1 that does not consider the direct effect of the velocity and radius in the consequent value. Model 2 is type- 1 first order linear model that considers the velocity as input variable. Model 3 is similar to model 2 except for the fact that the radius was adopted as an input. An additional test comprised of the inclusion of a hard constraint in the parameter estimation problem in order to avoid model predictions below 10\% (model 4) of the specialist decision. This constraint was proposed to ensure that the simulation results would not change more than $10 \%$ from the specialist assessment, even if the sum of squared errors increased. This approach means that the fuzzy model must guarantee an alignment with the direction suggested by the specialist.

While the type-2 fuzzy model structure was adopted in this work, the use of interval type-1 sets to represent the consequent parameters in a type-2 TSK model (with type-1 sets in the antecedents) would not have been a suitable choice since it provides the same output of a type-1 TSK model whose consequent parameters are the centers of the consequent sets of the interval type-2 TSK model (Mendel, 2001). In order to obtain a model better than all type-1 structures to control the hardware proposed (LEDs AFS), the type-2 fuzzy model considered in this work comprised the inclusion of an additional uncertainty level in the consequent of each rule (model output) through the adoption of parameters representing Gaussian (represented by ) (model 5) type-1 fuzzy sets. In this case, the mean and deviation of sets in all rules were considered in the model adjustment procedure. However, the results presented in Table 6 show that in this case the type- 2 structure proposed did not provided meaningful improvements in the model performance compared with the type- 1 structure (model 2) and model 5 was rejected as an alternative for LED control. The rejection of model 5 is also based on the greater simplicity of model 2 (type-1) in comparison with model 4 (type-2), mainly because of the small number of parameters (deviations of type-1 Gaussian fuzzy sets in the consequents are not considered).

All the fuzzy models listed in Table 6 comprise 8 rules (Table 5) and the output model parameters have specific values for each rule. For example, the number of parameters to be estimated in model 1 will be at most equal to 8 , and the specialist values presented in Table 4 were used as the initial guess in the parameter estimation algorithm. The performance of all these models was measured and compared through the average quadratic error. In all cases the intersection operation (type-1 fuzzy models) was performed applying the product operator

Table 6. Output models adopted and results.

\begin{tabular}{lclcccc}
\hline Model & Fuzzy type & Output model & \% Avg. quad error & Quadratic error & Std deviation & \% Avg. std deviation \\
\hline 1 & 1 & $y=a_{0}$ & 34.76 & $6,975.80$ & 437.65 & 86.18 \\
2 & 1 & $y=a_{0}+a_{1} \cdot V$ & 25.17 & $5,051.65$ & 316.88 & 62.40 \\
3 & 1 & $y=a_{0}+a_{1} \cdot R$ & 34.01 & $6,826.66$ & 427.63 & 84.21 \\
$4(*)$ & 1 & $y=a_{0}+a_{1} \cdot V$ & 27.23 & $5,465.95$ & 330.82 & 65.15 \\
5 & 2 & $\mathrm{y}=\left(a_{0} \pm \sigma\right) a_{1} \cdot V$ & 25.17 & $5,051.65$ & 316.88 & 62.40 \\
\hline
\end{tabular}

$(*)$ - Parameter estimation problem with hard constraint. 


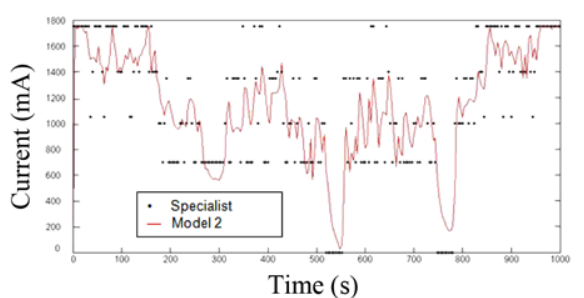

(a)

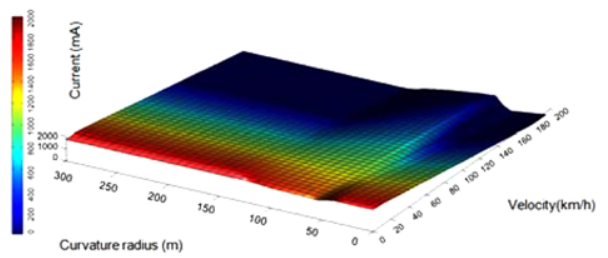

(b)

Figure 7. Comparison between specialist and model 2 (a) and surface generated from model 2 (b).

(Mendel, 2001). The operations used in the type-2 fuzzy model (meet and generalized centroid) were performed according to the extension principle (Karnik and Mendel, 2000).

Values of current output greater than of $1,750 \mathrm{~mA}$ (barrier) are saturated in this limit according to the system's hardware conditions (Figure 1).

Figure 7 shows the results of type-1 fuzzy models, with the lowest squared error in model 2. Although model 2 presents the best results, it still presents a high degree of variability and could lead to a degree of discomfort for the driver. In addition, another aspect worth mentioning is the light output. Despite the fact that the model presents good overall performance, in some cases it suggests current values below the specialist recommendation. This could result in further discomfort for the driver which could affect safety issues. A way to cope with this is to consider the adoption of hard constraints in the parameter estimation procedure. This approach was effectively implemented through model 4 which incorporates a hard constraint in which the values of current output should not vary more than $10 \%$ from the specialist's recommendation. The surface highlights the circumstances in which the LEDs will operate. Confirming the coherence of the model, Figure 7(b) presents a ramp showing how the current increases when the radius and speed decrease. The existence of a non uniform surface, with some abrupt drops in the current is expected and is associated to the nonlinearity of the fuzzy model and its intrinsic ability to interpolate different operational ranges.

Figure 8 shows the results obtained with model 4 (constraint of $10 \%$ in the parameter estimation procedure with fuzzy type-1). Compared with Figure 7(a), the model output values (total electrical current to LEDs) are closer to the specialist's suggestion, also providing a smoother surface and a better light distribution with more comfort for

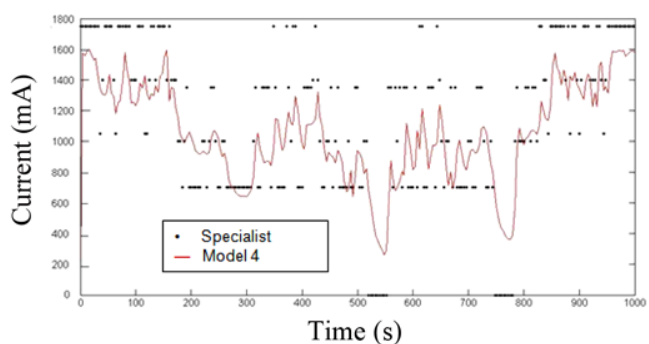

(a)

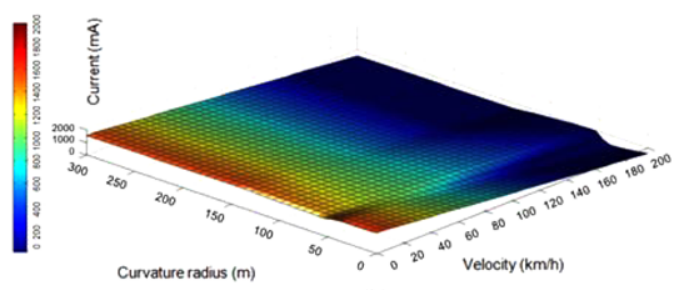

(b)

Figure 8. Comparison between specialist and model 4 (a) and surface generated from model 4 (b).

the driver on the road is obtained. Lower constraints were also tested, but a $10 \%$ restriction showed better performance due to the fact that it provided reduced fluctuations (small variance) and ensured the best outcome within a smaller quadratic error.

Considering global performance, model 4 would provide the best performance and consistency with respect to expert knowledge. In order to confirm that model 4 is also coherent according to its fuzzy surface, Figure 8(b) shows the model surface which presents the increase in current when the curves and speed decrease and the decrease in current when the speed and radius increase. It also shows a smoother surface when compared to model 2, specially around the $60 \mathrm{~m}$ radius where model 2 has an abrupt rupture generated by the fluctuation in the model. In this specific area, around $60 \mathrm{~m}$, it is very important to have a smooth distribution due to the fact that the AFS raises the luminous flux when compared to the system without AFS improving night-time driving, as can be seen in Table 3 .

Models 2 and 4 both have a better surface accommodation when compared to the surface generated from Table 4 (Figure 5) which is not only more abrupt but has many steps and imperfections on its surface. The surfaces of Figures 7 and 8 reveal more continuous behavior than the surface in Figure 5 (specialist surface) and is also more homogenous which helps to reduce the risk of possible distractions (or mistakes) during nighttime driving. The comparison between Figures $7 \sim 8$ and 5 is enough to justify the adoption of a modeling procedure suitable for the scenario described instead of using only the specialist rules.

\section{CONCLUSION}

This paper describes the development and application of a 
fuzzy rule-based system for modeling and predicting the amount of light to be added to the road to help the driver drive more safely during night time driving. The proposed modeling framework comprises a combination of expert knowledge and a parameter estimation procedure that enables the adjustment of the output models of each rule, considering different type-1 and type-2 fuzzy structures. More specifically, a specialist's engineering judgment can be appropriately incorporated in the fuzzy rule base according to the knowledge and experience on night time driving and environmental conditions.

Figure 8(b) shows the model surface which presents the increase in current when the curves and speed decrease and the decrease in current when the speed and radius increase. It also shows a smoother surface when compared to model 2 , specially around $60 \mathrm{~m}$ of radius where model 2 has an abrupt rupture generated by the fluctuation in the model. In this specific area, around $60 \mathrm{~m}$, it is very important to have a smooth distribution due to the fact that the AFS provides more luminous flux when compared to the system without AFS. Some of the models are satisfactory, but the best alternative is model 4 which considers a restriction that satisfy the specialist requirements and simultaneously provides a more comfortable light distribution for the driver. Although the model 4 does not give the minimum quadratic error, this provides output responses similar to the specialist contributing to night-time driving safer, while providing more light to the driver as well. The idea is that the specialist understands drivers' needs due to his knowledge.

Finally, it is important to highlight that the use of LEDs has certain advantages over halogen lighting, such as longer life, lower power consumption and color temperature among others. Further work will be done to improve the system and its algorithms.

ACKNOWLEDGEMENT-The authors acknowledge CAPES and CNPq (Brazilian federal research agencies) for their financial support. We also thank the Laboratory of Optical Proprieties at Universidade Federal da Bahia (Brazil) for their support and the support from the Ford Lighting Team located in Dearborn, Michigan (United States of America) and the Vehicle Evaluation Team at the test track in São Paulo (Brazil).

\section{REFERENCES}

AL (2009). Automotive Lighting Website. http://www.allighting.de.

Altinten, A., Erdgan, S., Hapglu, H. and Alpbaz, M. (2003). Control of a polymerization reactor by fuzzy control method with genetic algorithm. Comput. Chem. Eng., 27, 1031-1040.

Anderson, T., Sawyers, T. and Bell, M, (1998). Optimization of a fuzzy logic traffic signal controller by a multiobjective genetic algorithm. 9th Int. Conf. Road Transport Information and Control, 454, 186-190.

Babuška, R. and Verbruggen, H. B. (1996). An overview of fuzzy modeling for control. Control Eng. Practice, 4,
1593-1606.

Bellman, R. E. and Zadeh, L. A. (1970). Decision-making in a fuzzy environment. Management Science, 17, 141164.

Chen, B. and Liu, X. (2004). Reliable control design of fuzzy dynamic systems with time-varying delay. Fuzzy Sets and Systems, 146, 349-374.

Du, G.-N. and Zhu, Z.-Y. (2006). Modeling spatial vagueness based on type-2 fuzzy set. J. Zhejiang University SCIENCE A 1, 250-256.

Dubois, D. and Prade, H. (1978). Operations on fuzzy numbers. Int. J. Systems Science, 9, 613-626.

Dubois, D. and Prade, H. (1979). Operations in a fuzzyvalued logic. Information and Control, 43, 224-240.

Ekriasa, A., Eloholma, M., Halonen, L., Song, X., Zhang, X. and Wen, Y. (2008). Road lighting and headlights: Luminance measurements and automobile lighting simulations. Building and Environment, 43, 530-536.

EN (2003). Road Lighting-Part 3: Calculation of Performance. European Standard EN 13201-3, Publication 270-2003, Ref. No. EN 13201-3: 2003 E.

Feng, J., Li, J. and Yu, F. (2003). GA-based PID and fuzzy logic control for active vehicle suspension system. Int. J. Automotive Technology 4, 4, 181-191.

Gotoh, S. and Aoki, T. (1996). Development of active headlight. 15th Int. Conf. Enhanced Safety of Vehicles. Melbourne, Australia.

Hagras, H. A. (2004). A hierarchical type-2 fuzzy logic control architecture for autonomous mobile robots. IEEE Trans. Fuzzy Systems, 12, 524-539.

Hamm, M. (2002). Adaptive lighting functions history and future-Performance investigations and field test of user acceptance. SAE Paper No. 2002-01-0526.

Hara, T., Kuramochi, T., Ayama, M., Kojima, S. and Sato, T. (2001). Evaluation of AFS from Driver's Point of View, Progress in Automobile Lighting. Darmstadt. Germany' Technical University of Darmstadt. 397-402.

Hisdal, E. (1981). The IF-THEN ELSE statement and interval-values fuzzy sets of higher type. Int. J. ManMachine Studies, 15, 385-455.

Hogrefe, H. (2000). Adaptive front lighting systems for optimum illumination of curved roads, highway lanes and other driving situations. SAE Paper No. 2000-010431.

Ishiguro, K. and Yamada, Y. (2004). Control technology for bending mode AFS. SAE Paper No. 2004-01-0441.

Just Auto (2007). Annual Just-auto's Review of 2007 Management Briefing. Just Auto. Aroq Limited. UK.

Karnik, N. N. and Mendel, J. M. (1998). Introduction to type-2 fuzzy logic systems. Fuzzy Systems Proc. IEEE World Cong. Computational Intelligence. The 1998 IEEE Int. Conf., 2, 915-920.

Karnik, N. N. and Mendel, J. M. (2000). Operations on type-2 fuzzy sets. Int. J. Fuzzy Sets and Systems, 1, 327348.

King, P. J. and Mamdani, E. H. (1977). The application of 
fuzzy control systems to industrial processes. Automatica, 13, 235-242.

Kosonen, L. (2003). Multi-agent fuzzy signal control based on real-time simulation. World Cong. Intelligent Transport System, October, Transportation Research Part C: Emerging Technologies, 11, 389-403.

Hwang, J. and Song, C. (2005). Fuzzy estimation of vehicle speed using an accelerometer and wheel sensors. Int. J. Automotive Technology 6, 4, 359-365.

Hella (2009). Hella website. www.hella.de.

Li, L., Lin, W.-H. and Liu, H. (2006). Type-2 fuzzy logic approach for short-term traffic forecasting. Intelligent Transport Systems, IEEE Proc., 153, 33-40.

Liang, Q. and Mendel, J. M. (2000). Interval type-2 fuzzy logic systems: Theory and design. IEEE Trans. Fuzzy Systems, 8, 535-550.

Löwenau, J. P., Strob, M. H., Bernasch, J. H., Reich, F. M. and Rummel, A. H. (2001). Evaluation of adaptive light control in the BMW driving simulator. Proc. 2001 Driving Simulation Conf., Arcueil, France. INRETS.

Mendel, J. M. (2001). Uncertain Rule-based Fuzzy Logic Systems: Introduction and New Directions. PrenticeHall. London. UK.

Mendel, J. M. (2003). Fuzzy sets for words: A new beginning. The IEEE Int. Conf. Fuzzy Systems, 37-42.

Mendoza, O., Licea, G. and Melin, P. (2007). Modular neural networks and type-2 fuzzy logic for face recognition. Annual Meeting of the North American Fuzzy Information Processing Society, NAFIPS '07, 622-627.

Mizumoto, M. and Tanaka, K. (1976). Some properties of fuzzy sets of type-2. Information and Control, 31, 312340 .

Mizumoto, M. and Tanaka, K. (1981). Fuzzy sets of type-2 under algebraic product and algebraic sum. Fuzzy Sets and Systems, 5, 277-290.

Neumann, R. (2004). AFS halogen headlamp system: Experimental study and first field results. SAE Paper No. 2004-01-0439.

Neumann, R. (2006). LED front lighting - Optical concepts, styling opportunities and consumer expectations. SAE Paper No. 2006-01-0100.

Niittymäki, J. and Turunen, E. (2003). Traffic signal control n similarity logic reasoning. Fuzzy Sets and Systems, 133, 109-131.

Østergaard, J.-J. (1977). Fuzzy logic control of heat exchange process. M. M. Gupta, G. N. Saridis, B. R. Gaines (Eds.). Fuzzy Automata and Decision Process. North-Holland. Amsterdam. 285-320.

Pareek, U. and Kar, I. N. (2006). Estimating compressor discharge pressure of gas turbine power plant using type2 fuzzy logic systems. IEEE Int. Conf. Fuzzy Systems, 649-654.

Ren, Q., Qin, Z., Baron, L., Birglen, L. and Balazinski, M. (2007). Identification of rigid-body dynamics of robotic manipulators using type-2 fuzzy logic filter. Annual Meeting of the North American Fuzzy Information Processing Society, NAFIPS '07, 387-392.

Sala, A., Guerra, T. M. and Babuška, R. (2005). Perspectives of fuzzy systems and control. Fuzzy Sets and Systems, 156, 432-444.

Schmocker, J., Ahuja, S. and Bell, M. (2008). Multiobjective signal control of urban junctions- Framework and a London case study. Transportation Research Part $C, 16,454-470$.

Sivak, M., Schoettle, B., Flannagan, M. and Minoda, T. (2005). Optimal strategies for adaptive curve lighting. $J$. Safety Research, 36, 281-288.

Stern, D. (2008). Global Market Review of Automotive Lighting-Forecasts to 2013, 2008 Edn. Just Auto. Aroq Limited. UK.

Tahayori, H., Tettamanzi, A. G. B. and Degli Antoni, G. (2006). Approximated type-2 fuzzy set operations. IEEE Int. Conf. Fuzzy Systems, 1910-1917.

Thovutikul, S., Auephanwiriyakul, S. and Theera-Umpon, N. (2007). Microcalcification detection in mammograms using interval type-2 fuzzy logic system. IEEE Int. Fuzzy Systems Conf., FUZZ-IEEE 2007, 1-5.

UNECE (2008). UNECE website. http://www.unece.org/ trans/main/wp29/ wp29regs41-60.html

Wördenweber, B., Wallaschek, J., Boyce, P. and Donald D. Hoffman, D. (2007). Automotive Lighting and Human Vision. Springer Verlag. USA.

Yu, F., Feng, J. and Li, J. (2002). A fuzzy logic controller design for a vehicle ABS with a on-line optimized target wheel slip ratio. Int. J. Automotive Technology 3, 4, 165170.

Zadeh, L. A. (1975). The concept of a linguistic variable and its application to approximate reasoning - 1 , Information Sciences, 8, 199-249.

Zukauskas, A., Shur, M. and Caska, R. (2002). Introduction to Solid-State Lighting. Wiley Interscience. USA. 